\title{
The Not so Good, the Bad and the Ugly: Differential Bacterial Adhesion and Invasion Mediated by Salmonella PagN Allelic Variants
}

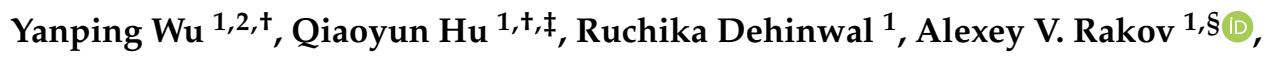 \\ Nicholas Grams ${ }^{1}{ }^{\circledR}$, Erin C. Clemens ${ }^{3, \|}$, Jennifer Hofmann ${ }^{3}$, Iruka N. Okeke ${ }^{3,4}{ }^{\oplus}$ and \\ Dieter M. Schifferli ${ }^{1, *(1)}$ \\ 1 Department of Pathobiology, University of Pennsylvania, School of Veterinary Medicine, \\ Philadelphia, PA 19104, USA; ypwu0902@163.com (Y.W.); huqiaoyun2020@163.com (Q.H.); \\ druchika@vet.upenn.edu (R.D.); rakovalexey@gmail.com (A.V.R.); gramsn@pennmedicine.upenn.edu (N.G.) \\ 2 College of Animal Science and Technology, College of Veterinary Medicine, Zhejiang Agriculture and \\ Forestry University, Hangzhou 311300, China \\ 3 Department of Biology, Haverford College, Haverford, PA 19041, USA; eclemen1@swarthmore.edu (E.C.C.); \\ hofmann.jen@gmail.com (J.H.); iruka.n.okeke@gmail.com (I.N.O.) \\ 4 Department of Pharmaceutical Microbiology, Faculty of Pharmacy, University of Ibadan, Ibadan 200284, \\ Oyo State, Nigeria \\ * Correspondence: dmschiff@vet.upenn.edu \\ + These authors contributed equally to this work. \\ $\ddagger$ Current address: Hunan Center for Animal Disease Control and Prevention, Changsha, Hunan, China. \\ $\S$ Current address: Somov Institute of Epidemiology and Microbiology, Vladivostok, Russia. \\ \| Current address: Swarthmore College, Swarthmore, PA, USA.
}

Received: 26 February 2020; Accepted: 28 March 2020; Published: 30 March 2020

\begin{abstract}
While advances in genomic sequencing have highlighted significant strain variability between and within Salmonella serovars, only a few protein variants have been directly related to evolutionary adaptation for survival, such as host specificity or differential virulence. The current study investigated whether allelic variation of the Salmonella adhesin/invasin PagN influences bacterial interaction with their receptors. The Salmonella enterica, subspecies enterica serovar Typhi (S. Typhi) allelic variant of PagN was found to bind significantly better to different enterocytes as well as to the extracellular matrix protein laminin than did the major Salmonella enterica, subspecies enterica serovar Typhimurium (S. Typhimurium) allele. The two alleles differed at amino acid residues 49 and 109 in two of the four predicted PagN surface loops, and residue substitution analysis revealed that a glutamic acid at residue 49 increased the adhesive and invasive properties of $S$. Typhi PagN. PagN sequence comparisons from 542 Salmonella strains for six representative S. enterica serovars and S. diarizonae further supported the role of glutamic acid at residues 49 and 109 in optimizing adhesion to cells and laminin, as well as for cell invasion. In summary, this study characterized unique residues in allelic variants of a virulence factor that participates in the colonization and invasive properties of different Salmonella stains, subspecies and serovars.
\end{abstract}

Keywords: Salmonella; S. Typhi; S. Typhimurium; S. diarizonae; PagN; adhesin; invasin; alleles; allelic variants

\section{Introduction}

Salmonella enterica subsp. enterica (S. enterica) is an entero-invasive bacterial pathogen that utilizes a type three secretion system (T3SS) encoded on the Salmonella pathogenicity island 1 (SPI-1) to invade intestinal epithelial cells. T3SS-driven uptake is particularly critical for intestinal S. enterica infections 
in mammals, as demonstrated with several experimental animal models [1]. S. enterica strains are differentiated by their flagella and O-antigens, which are highly variable and provide the basis for identification of over 2500 different serovars [2,3]. There is a direct correlation between the range of host adaptation of different $S$. enterica serovars with their levels of virulence and types of pathogenesis [4]. Serovars that are better adapted to a specific host species, such as the human-restricted S. enterica serovar Typhi (S. Typhi), are extremely pathogenic due to their ability to leave the intestines and spread hematogenously, resulting in sepsis. In contrast, serovars such as S. enterica serovar Typhimurium (S. Typhimurium) that have a broad host range are restricted to local invasion, and their containment by host inflammatory responses narrows their pathogenesis to gastrointestinal symptoms in humans. Finally, some Salmonella such as S. enterica subsp. diarizonae (S. diarizonae) are primarily associated with cold-blooded animals, and only rarely result in invasive diseases in sheep or humans [5,6].

Even though all Salmonella have and express SPI-1 genes [7], variations in the sequence of specific SPI-1 proteins and the unique repertoire of translocated SPI-1 effector proteins by different species, serovars and even strains within the same serovar has a direct impact on the invasion efficiency of different cell types [8-10]. Moreover, the diversity of S. enterica invasion levels for various cell types and host species can be partially attributed to variations in the regulation and export efficiencies of effector proteins through the SPI-1 T3SS [11]. In addition to the SPI-1 T3SS, cell invasion by S. enterica involves two outer membrane proteins (OMP), Rck and PagN, that have been designated adhesins-invasins due to their ability to promote both bacterial binding to host receptors and cellular uptake [12-14]. Rck has several adhesive properties: it binds to factor $\mathrm{H}$ to mediate bacterial resistance to complement and adheres to laminin and interacts with the epidermal growth factor receptor for a zipper mechanism of cell invasion [13,15-17]. Rck also contains a self-association motif that has the potential to mediate interbacterial attachment, as described for Hra1/Hek, an integral outer membrane protein of enteroaggregative Escherichia coli (E. coli) $[18,19]$. The adhesins/invasins Hra1/Hek, Hra2 and Tia of enteroaggregative or enterotoxigenic E. coli share homologies with membrane spanning domains of PagN, but their surface-exposed loops are less similar, alluding to variable binding affinities for different host receptors [18,20-22].

Unlike $r c k$, which is plasmid-encoded and absent in many virulent S. enterica serovars and strains, $\operatorname{pag} N$ is encoded on the bacterial chromosome and present in most, if not all, S. enterica. One study indicated that deletion of pagN in S. Typhimurium SL1344 grown under PhoPQ-activating conditions to inhibit SPI-1 gene transcription decreased bacterial adhesion and invasion of HT-29 cells [12]. In contrast, deletion of pagN in the $S$. Typhimurium strain LT2 did not impact adhesion to HT-29 or other cells [23], potentially due to the expression of other adhesins such as the type 1 fimbriae induced by the growth culture conditions. More surprisingly, despite the presence of both SPI-1 T3SS and type IV pili that can each independently mediate invasion, deletion of pag $N$ impacted both adhesion and invasion of HT-29 cells in a strain of S. Typhi [23,24]. Although the culture conditions used might not have activated functionally detectable T3SS expression, type IV pili were still functional, since a S. Typhi mutant lacking type IV pili affected invasion as much as the $\Delta p a g N$ mutant [23]. The latter result suggested that both PagN and the type IV pili contribute to $S$. Typhi invasion of cells. In addition, oral challenge of iron-overloaded Swiss Albino mice with wild type and pagN mutants of $S$. Typhi indicated that the former strain was more invasive and had increased lethality at an infectious dose of $2 \times 10^{7} \mathrm{CFU}$ and in competition assays. Finally, even though no differences were observed between the wild type and pagN mutant strains of S. Typhimurium [23], use of PagN from each serovar as an immunogen demonstrated some level of immune protection in mice challenged with the wild type strain from the corresponding serovar [23]. Thus, the role of PagN in the invasive properties of $S$. Typhi and $S$. Typhimurium remain somewhat controversial and studies of their function are likely impacted by the confounding effects of additional adhesins/invasins, different levels of PagN expression, and/or variations in the protein sequence of PagN.

Based on these results and our previous findings on FimH adhesin alleles in various S. enterica serovars and strains for host cell binding specificities $[25,26]$, we wondered whether allelic variation in 
PagN [10] modulates bacterial adhesion and invasion. Here we determined that the $S$. Typhi allelic variant of PagN provides significantly better bacterial binding and invasive efficiencies for different enterocytes and the extracellular matrix protein laminin, as compared to the major $S$. Typhimurium allele. To our surprise, PagN from S. enterica subsp. diarizonae was also significantly more adhesive and invasive than the major $S$. Typhimurium allele. Sequence comparison and functional analysis of substitutions as specific residue positions revealed that amino acids in two of the four PagN surface-exposed loops contributed to these phenotypes. These results further support the role of allelic variation of virulence factors in adjusting the level of pathogenic attributes among Salmonella subspecies and serovars.

\section{Materials and Methods}

\subsection{Bacterial Strain and Plasmid Constructions}

The bacterial strains and plasmids used in this study are described in Table 1 and all PCR primers are listed in Table S1. Unless stated otherwise, all the reagents were from MilliporeSigma (St. Louis, MO, USA). Bacteria were routinely grown in LB-Lennox media unless otherwise indicated. When appropriate, ampicillin $(200 \mu \mathrm{g} / \mathrm{mL})$ or kanamycin $(45 \mu \mathrm{g} / \mathrm{mL})$ was added to the growth medium. For plasmid constructions, the pagN genes were amplified from $S$. Typhimurium, S. Typhi and S. diarizonae genomic DNA by PCR with the Q5 High-Fidelity DNA Polymerase (New England Biolabs Inc., Ipswich, MA, USA). For PagN expression the allelic genes were cloned into AHT-inducible plasmid pRS1 using Gibson assembly with amplicon prepared from appropriate primers. Three site-directed substitution mutants were prepared by Gibson assembly of pRS1 and amplicons prepared with pagN external and internal primers for $S$. Typhimurium or $S$. Typhi, or amplicons prepared with mutagenic primers. A pagN-his fusion construct was prepared by insertion of a NdeI-HindIII restricted amplicon into pET22b. S. Typhimurium SL1344 sipB::aphA-3 mutant was prepared by P22 generalized transduction from strain DMS1507 [27,28] and SL1344 sipB::aphA-3 $\Delta$ pagN was constructed by Gibson assembly and allelic exchange as described [29-31]. All plasmid and strain constructs were confirmed by PCR and sequencing.

Table 1. Strains and plasmids.

\begin{tabular}{|c|c|c|}
\hline Strain & Genus, Species, Serovar and/or Relevant Genotype & Source \\
\hline AAEC189 & E. coli MM294 $\Delta$ lac recA endA $\Delta$ fim $\left(\mathrm{r}_{\mathrm{K}-} \mathrm{m}_{\mathrm{K}+}\right)$ & [32] \\
\hline BL21(DE3) & $\begin{array}{c}\text { E. coli str. B F- ompT gal dcm lon hsd } S_{B}\left(r_{B}{ }^{-} m_{B}{ }^{-}\right) \lambda(\mathrm{DE} 3 \\
{\left[\text { lacI lacUV5-T7p07 ind1 sam7 nin5]) }\left[\text { malB }^{+}\right]_{\mathrm{K}-12}\left(\lambda^{\mathrm{S}}\right)\right.} \\
\text { E. coli MG1655 }\end{array}$ & Novagen \\
\hline JKE201 & 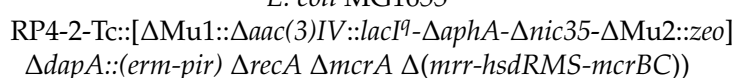 & {$[29,33]$} \\
\hline SL1344 & S. enterica subsp. enterica serovar Typhimurium & [34] \\
\hline S. Typhi & S. enterica subsp. enterica serovar Typhi 9,12,[Vi]:d:[Z66] & $\begin{array}{l}\text { Salmonella Reference collection C } \\
\text { (SARC) no2 }\end{array}$ \\
\hline S. diarizonae & S. enterica subsp. diarizonae $50: \mathrm{k}: \mathrm{z}$ & SARC no7 \\
\hline DMS1507 & 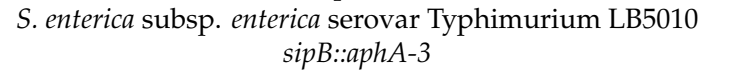 & {$[27,28]$} \\
\hline DMS1949 & SL1344 sipB $\triangle p a g N$ & This study \\
\hline Plasmid & Description & Source \\
\hline pMG81 & $\begin{array}{c}\text { Expression plasmid with tet } R \text { and tet } A \text { promoter } \\
\text { upstream } e n v Z, \mathrm{Ap}^{\mathrm{R}}\end{array}$ & Mark Goulian \\
\hline pRS1 & pMG81 & This study \\
\hline pDMS1973 & S. Typhi pagN in pRS1 & This study \\
\hline pDMS1974 & SL1344 pagN in pRS1 & This study \\
\hline pDMS2062 & S. diarizonae pag $N$ in $\mathrm{pRS} 1$ & This study \\
\hline pDMS2081 & SL1344 pagN $N_{\text {D49E }}$ in pRS1 & This study \\
\hline pDMS2082 & $\mathrm{SL}_{1344}$ pagN $N_{D 109 Q}$ in $\mathrm{pRS} 1$ & This study \\
\hline pDMS2083 & SL1344 pagN $_{D 109 E}$ in pRS1 & This study \\
\hline
\end{tabular}




\subsection{Protein Expression and Antibody Preparation}

The histidine-tagged PagN was expressed from the pET22b construct using the IPTG inducer and isolated by metal chelation chromatography as described previously [35]. A specific polyclonal antiserum against PagN-His was prepared in rabbits by using a conventional immunization protocol (Cocalico Biologicals Inc., Reamstown, PA, USA). The antiserum was adsorbed with E. coli BL21(DE3)/pET22b before use as described previously [36]. Briefly, $1 \mathrm{~mL}$ antiserum with $0.06 \%$ sodium azide was incubated with bacterial pellets from $10-\mathrm{mL}$ cultures grown overnight for $18 \mathrm{~h}$ at $4{ }^{\circ} \mathrm{C}$. After three adsorption cycles, the antiserum was filtered $(0.02 \mu \mathrm{m}$-pore-size) before use. PagN expression in E. coli AAEC189 for all the binding and invasion studies was induced with AHT by growing the bacteria overnight at $30^{\circ} \mathrm{C}$, or for $2 \mathrm{~h}$ at $37^{\circ} \mathrm{C}$, starting with $\log$ phase cultures $\left(\mathrm{A}_{600}=0.3\right)$. Comparable levels and bacterial surface expression of the three cloned PagN alleles were standardized by using various concentrations of inducers $(0.005-2 \mu \mathrm{g} / \mathrm{mL}$ AHT) for Western blot analysis and ELISA, as done previously [25]. Outer membrane proteins were prepared as described previously [37].

\subsection{Cell Cultures}

The human colonic cell line RKO (ATCC CRL2577) was cultured in Dulbecco's Modified Eagle Medium (DMEM; Invitrogen, Life Technologies) supplemented with $15 \%(v / v)$ heat inactivated fetal bovine serum (FBS) and antibiotics to a final concentration of $100 \mathrm{U} / \mathrm{mL}$ penicillin and $100 \mu \mathrm{g} / \mathrm{mL}$ streptomycin (Gibco, Life Technologies) [25]. The porcine cell line IPEC-J2 (DSMZ ACC 701) was cultured with 15\% heat inactivated FBS (Sigma-Aldrich), 1\% penicillin/streptomycin, 1\% insulin/transferrin/selenium (Gibco) and $5 \mathrm{ng} / \mathrm{mL}$ epidermal growth factor (Sigma) in DMEM/F-12/HAM $\left(1 / 1 / 1, v / v / v\right.$; Gibco). The cells were incubated at $37^{\circ} \mathrm{C}$ in a humid atmosphere with $5 \% \mathrm{CO}_{2}$.

\subsection{Bacterial Binding and Invasion Assays}

Both epithelial cell cultures grown to confluence in 24-well plates (Corning, CLS3596) were used for the binding assays with recombinant E. coli AAEC189 (E. coli $\Delta$ fim) with pRS1 plasmid constructs $\mathrm{s}$ expressing a Salmonella PagN allele. Bacteria were grown overnight, diluted $10^{-2}$ in LB broth with inducers (see above), incubated for 16-17 h, washed three times with PBS and diluted in DMEM to inoculate with a multiplicity of infection of 100 bacteria (in $0.25 \mathrm{~mL}$ ) to 1 enterocyte. Exact bacterial inoculum numbers were checked by standard CFU counts. The culture plates were centrifuged $(600 \mathrm{~g}, 5 \mathrm{~min})$ to initiate contact between the bacteria and the cells and incubated for $1 \mathrm{~h}$ at $37^{\circ} \mathrm{C}$ in $5 \% \mathrm{CO}_{2}$. To evaluate cell adhesion, the infected monolayers were washed thrice with PBS to remove non-associated bacteria and treated with $0.5 \%$ Triton X-100 to release and count the cell-associated bacteria by CFU enumeration. For the invasion assays, a standard gentamicin protection assay was performed [38]. Following a $1 \mathrm{~h}$ infection (see above), cells were incubated with medium containing gentamicin $(100 \mu \mathrm{g} / \mathrm{mL})$ for $90 \mathrm{~min}$ at $37{ }^{\circ} \mathrm{C}$ in $5 \% \mathrm{CO}_{2}$. After three washes with PBS, bacteria were released with Triton $\mathrm{X}-100$ and enumerated as described above. All experiments were done in triplicate wells and repeated at least thrice.

\subsection{Microscopy}

For fluorescence microscopy, E. coli strain AAEC189 carrying plasmids pRS1, pDMS1973 (pagN $\left.N_{\text {Ty }}\right)$, pDMS1974 $\left(\right.$ pag $\left._{\mathrm{Tm}}\right)$ or pDMS2062 $\left(p a g N_{\mathrm{di}}\right)$ were grown and induced for PagN expression as described above. The bacterial cells were deposited on slides, dried for $20 \mathrm{~min}$ and washed once with PBS. Bacteria were fixed with 4\% paraformaldehyde in PBS, pH 7.4, then labeled with adsorbed anti-PagN antisera (1:500), followed by anti-rabbit Alexa Fluor 480 (1:1000; Invitrogen, Life Technologies, Grand Island, NY, USA). Images were captured with a Coolsnap digital camera (Photometrics, Tucson, AZ, USA) mounted onto a Nikon Eclipse E600 microscope with Coolsnap version 1.2.0 software (Roper Scientific, Tucson, Arizona). 


\subsection{Binding to Extracellular Matrix Proteins}

Immuno Maxisorb plates with 96 wells (Nunc; Thermo Fisher Scientific, Rochester, NY, USA) were coated with $10 \mu \mathrm{g} / \mathrm{mL}$ of human collagen I, chicken collagen II, human collagen IV, bovine fibronectin, murine laminin or BSA (Sigma-Aldrich) in PBS at $4{ }^{\circ} \mathrm{C}$ overnight and then washed with PBS and blocked with PBS plus 1\% BSA for $2 \mathrm{~h}$. Binding of bacteria to laminin coated on plates was studied by using PagN-expressing E. coli. Bacteria grown and induced to make PagN were centrifuged, suspended in PBS to $10^{7} \mathrm{CFU}$ in $100 \mu \mathrm{L}$ and added to laminin coated wells. Bacteria harboring empty plasmid pRS1 were used as a control. After incubation for $1 \mathrm{~h}$ at $37^{\circ} \mathrm{C}$, unbound bacteria were removed by three washing cycles, anti-PagN antiserum (1:500) was added, followed by wash cycles and incubation with goat anti-rabbit HRP-conjugated antibody (1:2000). After three wash cycles, bound antibodies were detected by using the 1-Step Turbo TMB ELISA substrate (Thermo Fisher Scientific) followed by $2 \mathrm{M}$ sulfuric acid and measuring the absorbance at $450 \mathrm{~nm}$. For the binding inhibition assays, double dilutions of heparin or heparan sulfate $(400-0.4 \mu \mathrm{g} / \mathrm{mL})$ were incubated with bacteria for $1 \mathrm{~h}$ and the mixtures were added to the laminin-coated wells for further processing as described above.

\subsection{Bacterial Genomes and PagN Sequences}

Salmonella genomic sequences from 497 previously studied S. enterica from 6 serovars with two biovars (serovars Typhi, Dublin, Choleraesuis, Typhimurium, Enteritidis, Newport and Gallinarum, with biovars Gallinarum and Pullorum for the latter serovar) and from 45 confirmed S. diarizonae (Table S2) were obtained from NCBI RefSeq database or assembled from NCBI SRA, EBI ENA and Wellcome Sanger Institute repositories as described [10]. The genomes were used to determine their encoded PagN sequences. The genomes of 13 incorrectly serotyped $S$. diarizonae strains were detected with SISTR (their corrected serovar attribution was added to Table S2) and not analyzed for PagN [39]. Protein alignments were done with Megalign, DNASTAR Lasergene (Madison, WI, USA).

\subsection{PagN Structure Analysis}

The 3D-structural model of PagN was predicted by using the corresponding sequence from S. Typhimurium LT2 and I-TASSER $[40,41]$. Among the five best predicted models, the 3rd model was chosen to be shown in Figure 1B (Protean 3D, DNASTAR Lasergene), with an overall ERRAT quality factor of 84.4 [42], in agreement with a published model for $S$. Typhi PagN [43]. The five best I-TASSER predicted S. arizonae PagN structures essentially overlapped with the ones of S. Typhimurium and S. Typhi.

\subsection{Statistical Analysis}

Student's non-paired $t$ test (two tailed) was used with Prism 8 (GraphPad Software, San Diego, CA, USA) to calculate statistical significance for all the binding assays $\left({ }^{*} p<0.05,{ }^{* *} p<0.01,{ }^{* * *} p<0.001\right.$ and $\left.{ }^{* * * *} p<0.0001\right)$.

\section{Results and Discussion}

PagN of $S$. Typhimurium and $S$. Typhi had similar sequences with two to three substitutions in predicted surface-exposed loops 1 and 2 (Figure 1). Since PagN of both strains act as adhesins and invasins for human intestinal epithelial cells, we wondered whether these allelic variants differentially impacted these properties. To ensure consistent and comparable expression of PagN, we cloned each gene into inducible expression plasmids to make pDMS1973 and pDMS1974 for the expression of PagN from S.Typhi ( $\left.p a g N_{\mathrm{Ty}}\right)$ and $S$. Typhimurium $\left(p a g N_{\mathrm{Tm}}\right)$ respectively. Western blot analysis of outer membrane preparations from E. coli AAEC89 carrying one of these two plasmid constructs detected bands specific for each PagN allele (Figure 2A). 
A

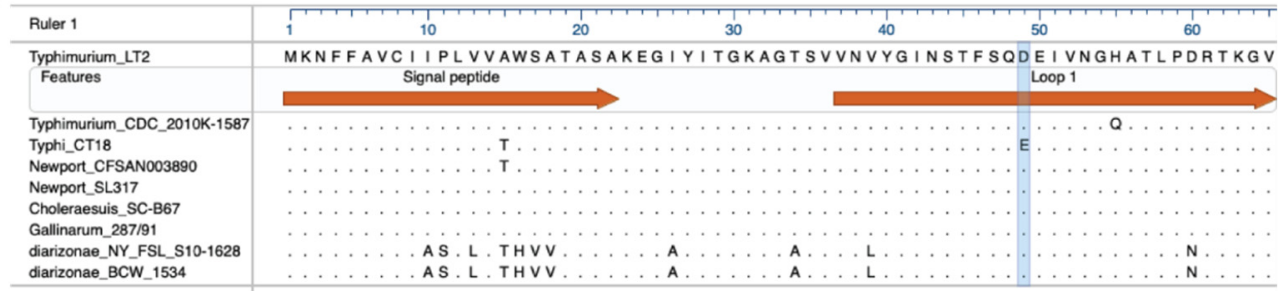

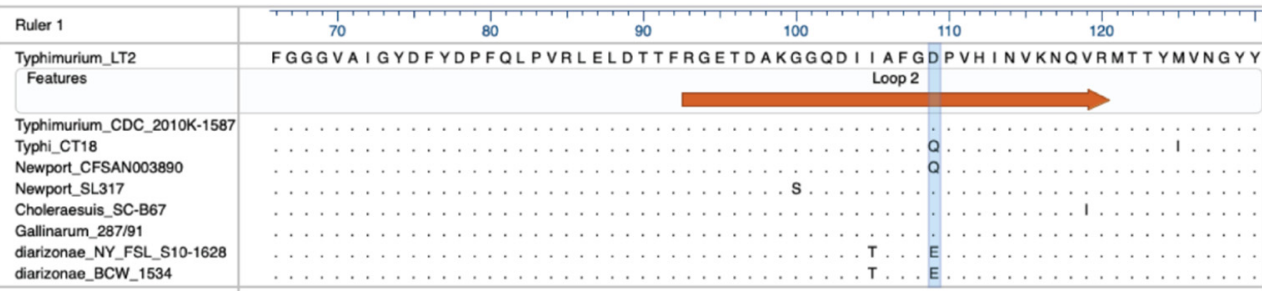

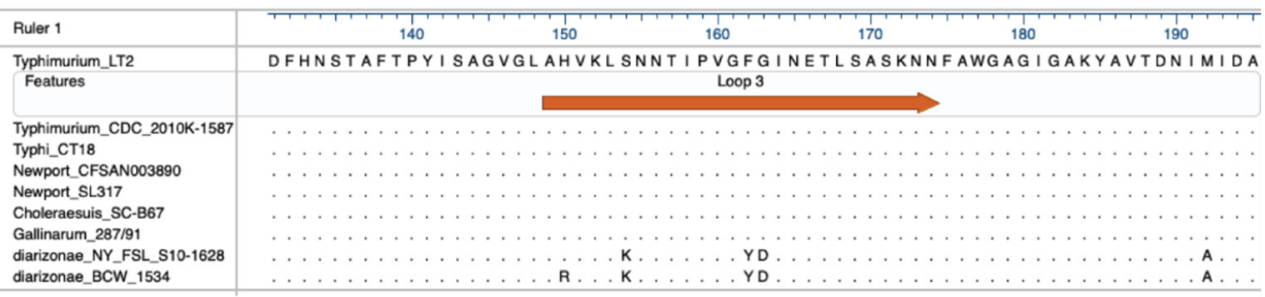

\begin{tabular}{|c|c|}
\hline Ruler 1 & 220 \\
\hline \multirow{2}{*}{$\begin{array}{l}\text { Typhimurium_LT2 } \\
\text { Features }\end{array}$} & SYKY INAGKVSISKNHYAGDEHTAYDADTKAASNDFMLGITYAF \\
\hline & Loop 4 \\
\hline Typhimurium_CDC_2010K-1587 & 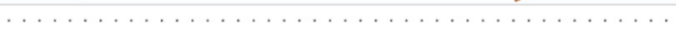 \\
\hline Typhi_CT18 & 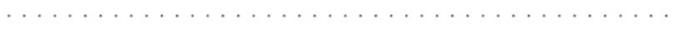 \\
\hline Newport_CFSAN003890 & - \\
\hline Newport_SL317 & 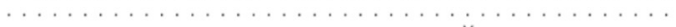 \\
\hline Choleraesuis_SC-B67 & 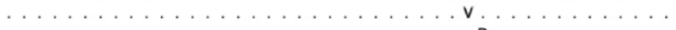 \\
\hline Gallinarum_287/91 & $\ldots \ldots \ldots \ldots \ldots \ldots \ldots \ldots \ldots \ldots \ldots \ldots$ \\
\hline diarizonae_NY_FSL_S10-1628 & 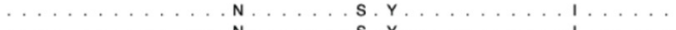 \\
\hline diarizonae_BCW_1534 & 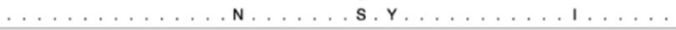 \\
\hline
\end{tabular}

B

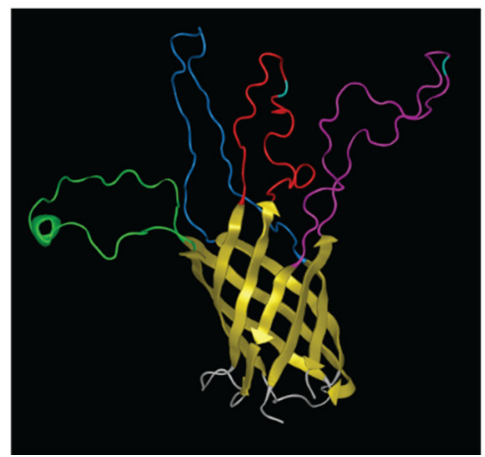

Figure 1. PagN sequences and predicted structure. (A) Alignment of PagN alleles from different Salmonella strains (Table S3) [10] each representing N strains from one or more serovars, or clusters within serovars or subspecies with the same sequence in the surface loops of mature PagN; $S$. Typhimurium_LT2 $(n=68)$ with same sequence for $S$. Enteritidis $(n=74), S$. Pullorum $(n=29), S$. Dublin $(n=74)$, and a cluster of Newport strains $(n=25) ; S$. Typhimurium_CDC_2010k-1587 $(n=7) ; S$. Typhi_CT18 $(n=75)$; S. Newport_CFSAN003890 $(n=23) ;$ S. Newport_SL317 $(n=22) ;$ S. Choleraesuis_SC-B67 $(n=74)$; S. Gallinarum 287/91 $(n=23)$; S. diarizonae NY_FSL_S10-1628 $(n=3)$ and S. diarizonae_BCW_1534 $(n=45)$. (B) $S$. Typhimurium mature PagN model predicted with I-TASSER. Color codes are for the membrane spanning beta-barrel (yellow), the periplasmic loops (grey), surface-exposed loop 1 (purple) with residue 49 (turquoise), loop 2 (red) with residue 109 (turquoise), loop 3 (green) and loop 4 (dark blue). 


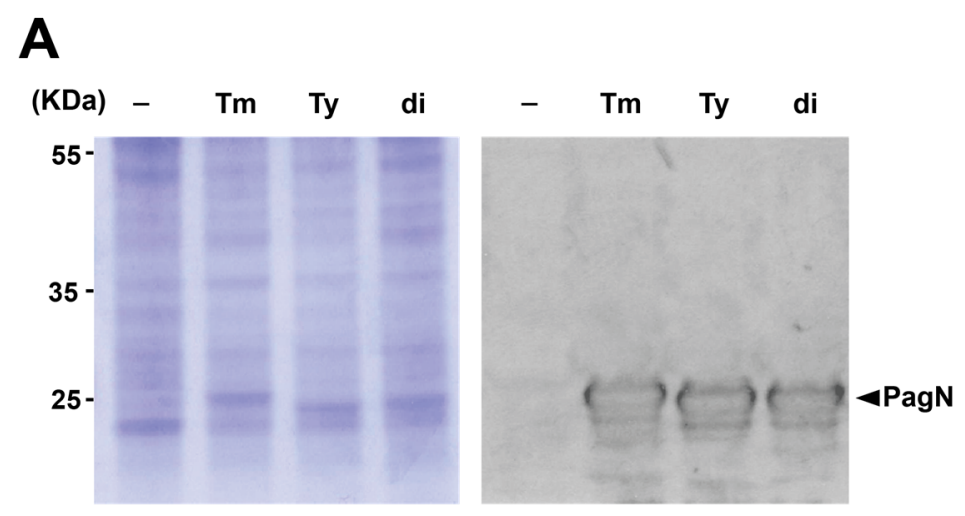

B
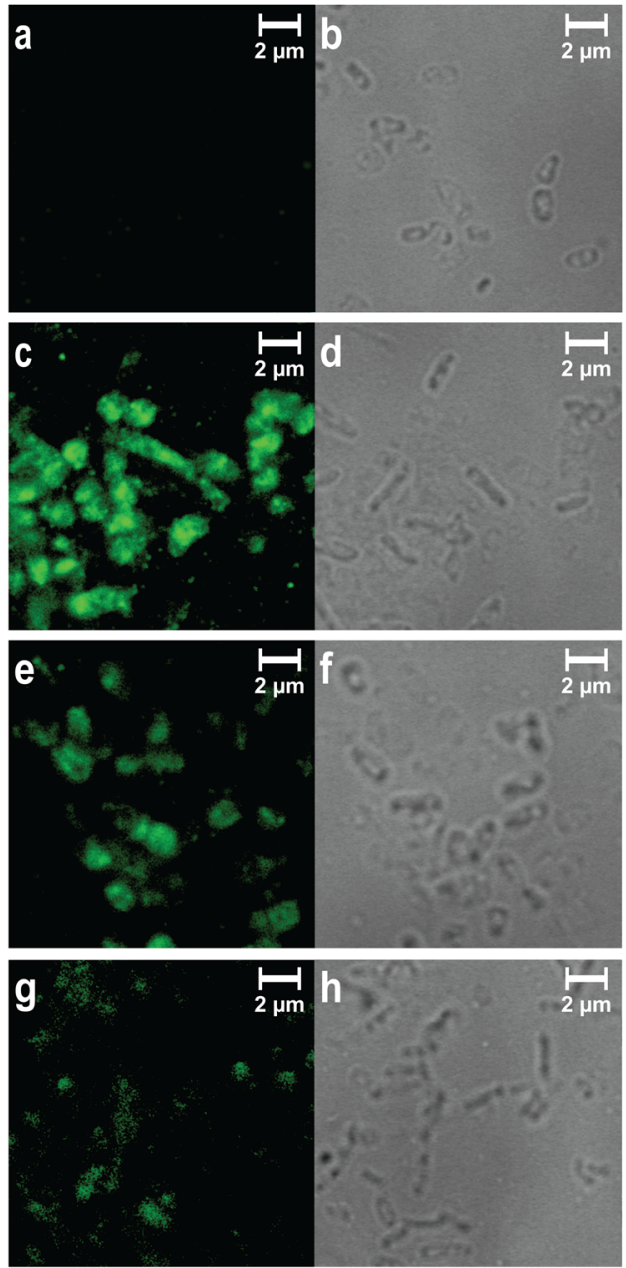

Figure 2. Expression of pagN alleles from S. Typhimurium, S. Typhi and S. diarizonae. (A) Expression of PagN in E. coli AAEC189 transformed with pRS1 (empty vector, -), pDMS1973 (pagN $N_{\text {Ty }}$ ), pDMS1974 $\left(\right.$ pag $\left.N_{\mathrm{Tm}}\right)$ or pDMS2062 $\left(p a g N_{\mathrm{di}}\right)$ was induced by AHT $(0.2-0.4 \mu \mathrm{g} / \mathrm{mL})$ for $2 \mathrm{~h}$ at $37^{\circ} \mathrm{C}$. Isolated outer membrane proteins analyzed by SDS-PAGE, followed by Coomassie blue staining or western blotting with anti-PagN antisera showed a clear band for PagN expression at $25 \mathrm{kDa}$. (B) Visualization of PagN surface expression in E. coli AAEC189 expressing the allele from S. Typhimurium (c,d), S. Typhi (e,f), S. diarizonae $(\mathbf{g}, \mathbf{h})$ or empty vector as negative control $(\mathbf{a}, \mathbf{b})$. Phase-contrast microscopy (right) and fluorescence microscopy (left) were used to detect bacteria labeled with anti-PagN antisera, followed by Alexa Fluor 488-conjugated anti-rabbit IgG. 
Moreover, both alleles of PagN were detectable on the bacterial surface by immunofluorescence (Figure 2B, panel a to f) and ELISA (not shown). Not surprisingly, the strongest signal was observed for the $S$. Typhimurium PagN allele, as this was the immunogen used to prepare the antiserum.

The E. coli strain AAEC189 lacks adhesive type 1 fimbriae, providing us with a bacterial context in which to study PagN-mediated bacterial binding and cellular uptake free of known Salmonella adhesins and invasins. Using both RKO and IPEC-J2 enterocytes, we found that both PagN $\mathrm{Ny}_{\mathrm{Ty}}$ and PagN $\mathrm{N}_{\mathrm{Tm}}$ mediated bacterial binding and invasion, as previously reported for ovarian hamster epithelial-like CHO-K1 cells and human colonic HT-29 cells [12,23]. More importantly, comparisons of the two alleles indicated that Pag $\mathrm{N}_{\mathrm{Ty}}$ was more efficient than PagN $\mathrm{N}_{\mathrm{Tm}}$ for both cell adhesion and invasion (Figure 3A), supporting an allelic variant effect on a virulence property. Additional studies with a S. Typhimurium $p a g N$ and $\operatorname{sipB}$ (essential SPI-1 translocon subunit) deletion mutant complemented with different PagN-expressing plasmids did not show significant different levels of adhesion/invasion of RKO cells (data not shown), suggesting a dominant phenotype due to the expression of one or more other Salmonella adhesin(s) and invasin(s) under the used growth conditions. Thus, the use of E. coli with a controlled expression system allowed us to bypass the masking effect of additional Salmonella adhesins/invasins differentially expressed in different serovars and environmental growth conditions and identify functional differences due only to sequence variations of PagN alleles [44,45].

A

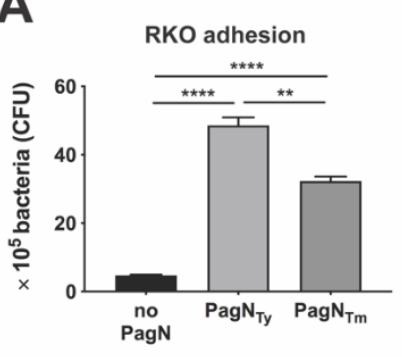

IPEC-J2 adhesion

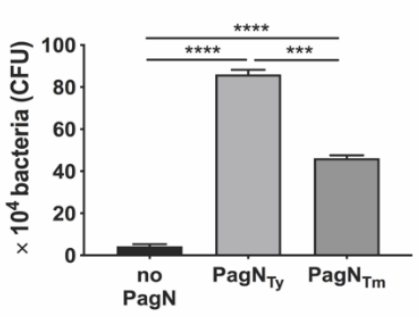

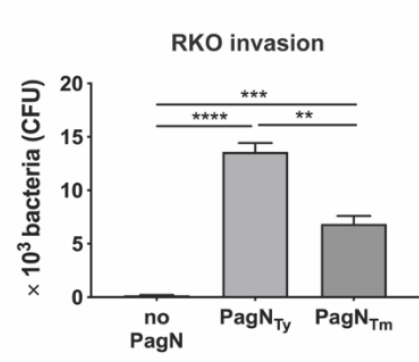

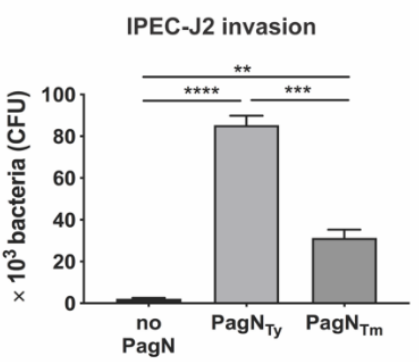

B
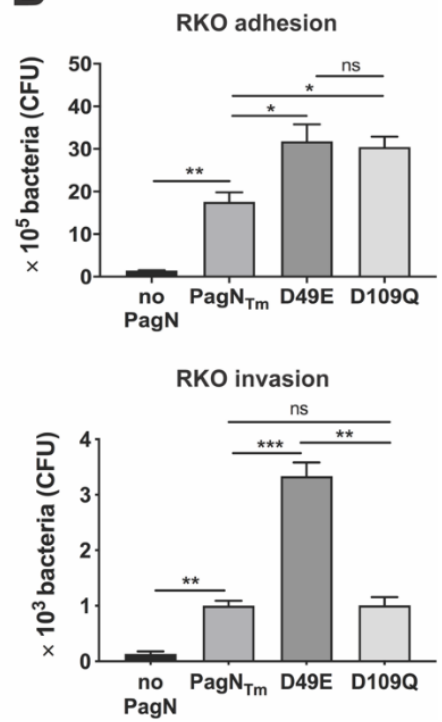

Figure 3. Binding and uptake of E. coli AAEC189 to intestinal epithelial cells mediated by PagN expression. (A) Adherence and invasion of E. coli AAEC189 with empty vector pRS1 (no pagN), pDMS1973 or pDMS1974 to express PagN $\mathrm{N}_{\text {Ty }}$ or PagN $\mathrm{Pm}_{\mathrm{Tm}}$ respectively, to human (RKO) and porcine (IPEC-J2) was analyzed by incubating $3 \times 10^{7} \mathrm{CFU} / \mathrm{mL}$ bacteria at a MOI of 100 for $60 \mathrm{~min}$ for adherence and another $90 \mathrm{~min}$ for invasion assays. Bacteria expressing $\mathrm{PagN}_{\mathrm{Ty}}$ bound and invaded significantly better than the bacteria expressing $\operatorname{PagN}_{\mathrm{Tm}}(p<0.01-0.001)$. (B) Adherence of E. coli AAEC189 expressing PagN $\mathrm{N}_{\mathrm{Tm}}$ to RKO cells was significantly enhanced $(p<0.05)$ when $\operatorname{PagN}_{\mathrm{Tm}}$ was mutated from aspartate to glutamate (at site 49, D49E) with pDMS2081 or to glutamine (at site 109, D109Q) with pDMS2082, whereas invasion of epithelial cells was affected only by the D49E substitution $(p<0.001)$, but not by the D109Q substitution. Data represent one of three separate and reproducible experiments each with triplicate data expressed as mean \pm SEM (ns is for not significant, ${ }^{*} p<0.05,{ }^{* *} p<0.01$, *** $p<0.001$ and $\left.{ }^{* * * *} p<0.0001\right)$.

PagN sequence alignments for 52-75 strains for each of the seven S. enterica serovars, including S. Typhimurium [10], highlighted that all PagN had aspartic acid residues at position 49 and 109, with the exceptions of $S$. Typhi that had a glutamic acid at position 49 , and a group of $S$. Newport strains that had a glutamine at position 109 (Figure 1A). Since the allelic PagN proteins of all strains of 
S. Typhi and $S$. Typhimurium varied at these two positions and were each predicted to be located in a different bacterial surface loop (Figure 1B), we investigated their relative involvement in bacterial adhesion and invasion. For this, we generated PagN $\mathrm{N}_{\mathrm{Tm}}$ with a D49E or a D109Q substitution and expressed them in E. coli strain AAEC189. Although both mutated PagN mediated better bacterial binding to RKO cells than did PagN $\mathrm{Tm}_{\mathrm{T}}$, only the D49E substitution in PagN had a significantly stronger effect on bacterial invasion (Figure 3B). This result highlighted the contribution of the $S$. Typhi glutamic acid at position 49 for the improved interaction of PagN with a host cell receptor to promote bacterial uptake. This result is consistent with a role for the PagN allele in the increased pathogenicity of $S$. Typhi relative to $S$. Typhimurium pathogenesis following human infection, in agreement with the former serovar's hematogenous bacterial spreading in humans resulting frequently in sepsis, in contrast to the latter serovar and its pathogenesis that is usually contained in the gastro-intestinal organs.

Other than subsp. enterica, most subsp. of S. enterica are associated with cold-blooded animals. Therefore, we expected that a construct expressing the PagN allele of these non-enterica subspecies would neither bind nor efficiently invade human RKO cells and therefore would serve as a negative control. Thus, we cloned the $S$. diarizonae $\mathrm{PagN}\left(\mathrm{PagN}_{\mathrm{di}}\right)$ in the same inducible expression plasmid used for the two other PagN alleles to make pDMS2062 and confirmed protein production (Figure 2A) and surface expression (Figure 2B, panels $\mathrm{g}$ and $\mathrm{h}$ ). To our surprise, E. coli making PagN $\mathrm{N}_{\mathrm{di}}$ were significantly more adhesive and invasive than the ones expressing $\mathrm{PagN}_{\mathrm{Tm}}$ (Figure $4 \mathrm{~A}$ ).
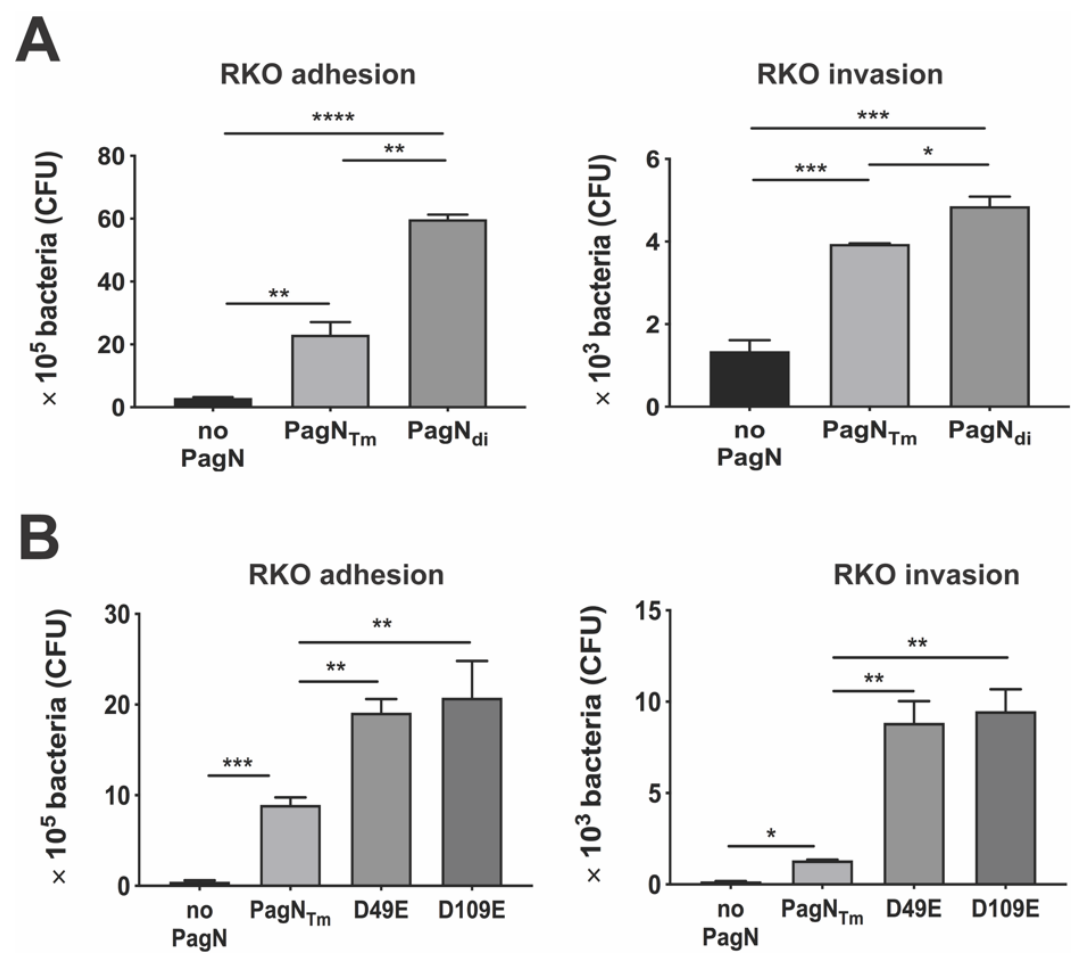

Figure 4. Binding and uptake of E. coli expressing PagN from non-enterica allelic variants. (A) Adhesion and invasion of E. coli AAEC189 expressing pagN allele from $S$. diarizonae $\operatorname{PagN}\left(\mathrm{PagN}_{\mathrm{di}}\right)$ and $S$. Typhimurium PagN $\left(\operatorname{PagN}_{\mathrm{Tm}}\right)$ to RKO cells showed the bacteria expressing $P$ Pag $\mathrm{N}_{\mathrm{di}}$ binds significantly better than $\operatorname{PagN}_{\mathrm{Tm}}(p<0.01)$ and was slightly more invasive $(p<0.05)$. (B) A substitution of aspartate to glutamate at site 109 in S. Typhimurium PagN (D109E) increased both binding and invasion of E. coli into RKO cells as compared to the bacteria expressing $\operatorname{PagN}_{\mathrm{Tm}}(p<0.01)$. Data represent one of three separate and reproducible experiments, each with triplicate data expressed as mean $\pm \operatorname{SEM}\left({ }^{*} p<0.05,{ }^{* *} p<0.01,{ }^{* * *} p<0.001\right.$ and $\left.{ }^{* * * *} p<0.0001\right)$.

PagN alignments of 48 available strains of S. diarizonae highlighted that they all had an aspartic acid at position 49, like most evaluated subsp. enterica serovars (Typhi being the exception, Figure 1A). 
In contrast, unlike the subsp. enterica serovars, all $\mathrm{PagN}_{\mathrm{di}}$ carried a glutamic acid at position 109, suggesting that a D109E substitution at this position would be functionally significant. In support, a D109E substitution in the $S$. Typhimurium PagN (pDMS 2083) improved not only bacterial binding, but also bacterial uptake by RKO cells (Figure 4B), indicating that glutamic acid in this position optimizes the function of PagN as both an adhesin and invasin. Whether other substitutions in $\mathrm{PagN}_{\mathrm{di}}$, such as the arginine or lysine at position 150 and 154 in loop 3 (Figure 1) modulate bacterial invasion remains to be determined. Like $S$. Typhi, S. diarizonae have type IVB pili involved in intestinal cell invasion, raising the possibility that the concomitant increased binding property of the S. diarizonae PagN allele contributes to the reported increased bacterial virulence in humans under specific conditions [5]. Taken together, the results showed that PagN alleles with a glutamic acid residue at either 49 or 109 improves bacterial binding and/or invasiveness, possibly due to the more ionizable and long chain characteristics of glutamic acid relative to aspartic acid and glutamine.

In addition to promoting bacterial attachment to cells, many bacterial adhesins interact with various host glycoprotein of the extracellular matrix, as exemplified by the ability of $\mathrm{PagN}_{\mathrm{Ty}}$ to bind to laminin [23,46-48]. S. enterica may encounter laminin either on intestinal areas denuded of epithelial cells (e.g., extrusion zones at villi tips) or in the subepithelial space after invading enterocytes and crossing the intestinal epithelial layer. Notably, the low $\mathrm{pH}$ and magnesium concentrations in Salmonella-containing vacuoles (SCV) of invaded cells induce the PhoPQ two-component system responsible for PagN expression [49-51]. Thus, whereas the S. enterica SPI-1 system is induced in the intestinal environment and used for enterocyte invasion, expression of PagN in SCV (where SPI-1 is largely repressed) may prepare Salmonella for future cycles of cell invasion after escape from intestinal epithelial cells. Our side-by side comparison of $\mathrm{PagN}_{\mathrm{Ty}}{ }^{-}, \mathrm{PagN}_{\mathrm{Tm}^{-}}$and $\mathrm{PagN}_{\mathrm{di}}$-expressing E. coli showed that all three bacteria adhered significantly to laminin, albeit the former better than the latter two (Figure 5A). None of the bacteria bound to collagen I, II or IV or fibronectin (not shown).

A

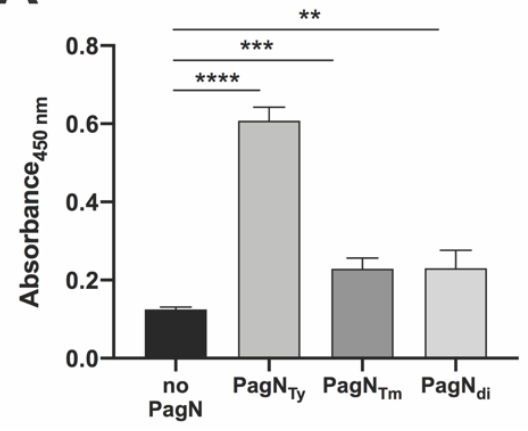

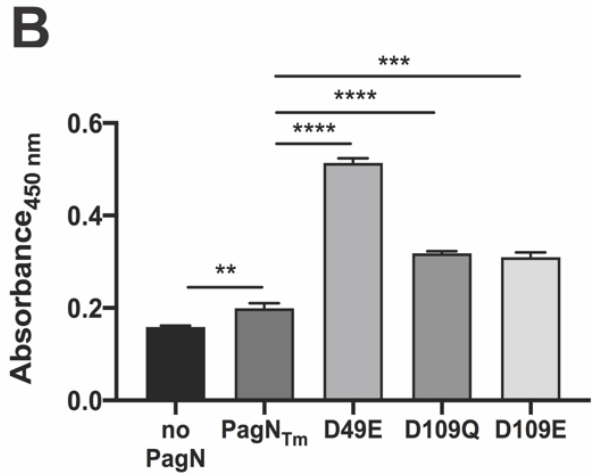

Figure 5. Binding of PagN allelic variants to murine laminin. (A) The PagN $\mathrm{Ty}_{\mathrm{y}}$-expressing bacteria bound best to laminin. E. coli AAEC189 expressing PagN $\mathrm{Tm}_{\mathrm{T}}, \mathrm{PagN}_{\mathrm{Ty}}, \mathrm{PagN}_{\mathrm{di}}$ or containing empty vector (no $\operatorname{pagN}$ ) were incubated with microtiter wells coated with murine laminin. Bacterial binding was detected by an ELISA-method with anti-PagN antiserum. (B) All substitution mutants bound better to laminin than bacteria expressing PagN $\mathrm{Nm}_{\mathrm{Tm}}$, with the mutant expressing glutamic acid at position 49 of $\mathrm{PagN}_{\mathrm{Tm}}$ (D49E) binding best $(p<0.0001)$. Data represent one of three separate and reproducible experiments, each with triplicate data expressed as mean $\pm \operatorname{SEM}\left({ }^{* *} p<0.01\right.$, $^{* * *} p<0.001$ and $\left.{ }^{* * * *} p<0.0001\right)$.

To determine the potential role of PagN residue 49 and 109 in $\mathrm{PagN}_{\mathrm{Ty}}$ binding to laminin, we tested the three mutants described above. As for the intestinal epithelial cell binding results, bacteria with a glutamic acid at position 49 of $\mathrm{PagN}_{\mathrm{Tm}}$ (D49E) bound significantly better to laminin than did bacteria with PagN $\mathrm{Tm}_{\mathrm{m}}$ (Figure 5B). Glutamic acid or glutamine at position 109 of $\mathrm{PagN}_{\mathrm{Tm}}$ (D109E and D109Q) also increased binding, albeit less efficiently.

Since both PagN and heparin bind to laminin [52], we next determined whether heparin or heparan sulfate could inhibit PagN-mediated bacterial binding to laminin. In contrast to the reported inhibitory 
effect of heparin on PagN-mediated invasion of CHO-K1 cells [53], neither heparin nor heparan sulfate at concentrations as high as $400 \mu \mathrm{g} / \mathrm{mL}$ interfered with the binding of $\mathrm{PagN}_{\mathrm{Ty}}$-expressing bacteria to laminin (data not shown). These combined results suggested that PagN and heparin bind to different laminin sites and that the invasion of CHO-K1 cells by PagN-expressing bacteria is laminin-independent. Thus, our studies highlight the independent adhesive properties of PagN in binding to either intestinal cells or the extracellular matrix protein laminin, two relevant targets for Salmonella host invasion.

In summary and together with our previous studies on variants of fimbrial adhesins $[25,26,54]$, this study on PagN, a Salmonella adhesin/invasin, further supports the importance of protein sequence allelic variants in virulence properties [55], including pathogenic properties such as adhesion and invasion [4].

Supplementary Materials: The following are available online at http://www.mdpi.com/2076-2607/8/4/489/s1.

Author Contributions: Conceptualization, D.M.S. and I.N.O.; methodology, D.M.S. and I.N.O.; software, A.V.R. and D.M.S.; validation, D.M.S. and R.D.; formal analysis, Y.W., Q.H., R.D., N.G.; investigation, Y.W., Q.H., R.D., N.G., E.C.C. and J.H.; resources, D.M.S. and I.N.O.; data curation, R.D. and D.M.S.; writing-original draft preparation, D.M.S.; writing-review and editing, D.M.S.; visualization, R.D., Y.W., Q.H.; supervision, D.M.S. and I.N.O.; project administration, D.M.S. and I.N.O.; funding acquisition, D.M.S. and I.N.O. All authors have read and agreed to the published version of the manuscript.

Funding: This research was funded by USDA National Institute of Food and Agriculture grant 2013-67015-21285, NIH/NIAID grants AI117135 and AI139982, and the PennVet Center for Host-Microbial Interactions to DMS, and NSF RUI awards MCB 0948460 and MCB1329248 to INO. QH was supported by a grant from the China Scholarship Council and YW was supported by a grant from the International Cooperative Research and Exchange Program of Zhejiang University. The funding bodies had no role in the design of the study and collection, analysis and interpretation of data and in writing the manuscript.

Acknowledgments: We thank all the contributors for publicly sharing their Salmonella genomic data through the National Center for Biotechnology Information, European Bioinformatics Institute and Wellcome Sanger Institute Salmonella genome projects. We thank Mark Goulian for plasmid pMG81, Olivier Cunrath and Dirk Bumann for E. coli strain JKE201 and Gibson assembly protocols, and Leslie King for proofreading the manuscript. The opinions expressed in this manuscript are solely the responsibility of the authors and do not necessarily represent the official views and policy of the National Institutes of Health.

Conflicts of Interest: The authors declare no conflict of interest. The funders had no role in the design of the study; in the collection, analyses or interpretation of data; in the writing of the manuscript, or in the decision to publish the results.

\section{References}

1. Tsolis, R.M.; Xavier, M.N.; Santos, R.L.; Baumler, A.J. How to become a top model: Impact of animal experimentation on human Salmonella disease research. Infect. Immun. 2011, 79, 1806-1814. [CrossRef]

2. Alikhan, N.F.; Zhou, Z.; Sergeant, M.J.; Achtman, M. A genomic overview of the population structure of Salmonella. PLoS Genet. 2018, 14, e1007261. [CrossRef]

3. Grimont, P.A.D.; Weill, F.-X. Antigenic Formulae of the Salmonella Serovars; WHO Collaborating Center for Reference and Research on Salmonella; Institut Pasteur, 28 rue du Dr. Roux: Paris, France, 2007; pp. 1-166.

4. Yue, M.; Schifferli, D.M. Allelic variation in Salmonella: An underappreciated driver of adaptation and virulence. Front. Microbiol. 2014, 4, 419. [CrossRef]

5. Giner-Lamia, J.; Vinuesa, P.; Betancor, L.; Silva, C.; Bisio, J.; Soleto, L.; Chabalgoity, J.A.; Puente, J.L.; Salmonella, C.N.; Garcia-Del Portillo, F. Genome analysis of Salmonella enterica subsp. diarizonae isolates from invasive human infections reveals enrichment of virulence-related functions in lineage ST1256. BMC Genomics 2019, 20, 99. [CrossRef]

6. Uelze, L.; Borowiak, M.; Deneke, C.; Jacobs, C.; Szabo, I.; Tausch, S.H.; Malorny, B. First complete genome sequence and comparative analysis of Salmonella enterica subsp. diarizonae serovar 61:k:1,5,(7) indicates host adaptation traits to sheep. Gut Pathog. 2019, 11, 48. [CrossRef]

7. Bäumler, A.J.; Tsolis, R.M.; Ficht, T.A.; Adams, L.G. Evolution of host adaptation in Salmonella enterica. Infect. Immun. 1998, 66, 4579-4587. [CrossRef]

8. Hopkins, K.L.; Threlfall, E.J. Frequency and polymorphism of sopE in isolates of Salmonella enterica belonging to the ten most prevalent serotypes in England and Wales. J. Med. Microbiol. 2004, 53, 539-543. [CrossRef] 
9. Heffron, F.; Niemann, G.; Yoon, H.; Kidwai, A.; Brown, R.N.E.; McDermott, J.D.; Smith, R.; Adlkins, J.N. Salmonella-Secreted Virulence Factors. In Salmonella: From Genome to Function; Porwollik, S., Ed.; Caister Academic Press: Norfolk, UK, 2011; pp. 187-223.

10. Rakov, A.V.; Mastriani, E.; Liu, S.L.; Schifferli, D.M. Association of Salmonella virulence factor alleles with intestinal and invasive serovars. BMC Genom. 2019, 20, 429. [CrossRef]

11. Elhadad, D.; Desai, P.; Grassl, G.A.; McClelland, M.; Rahav, G.; Gal-Mor, O. Differences in Host Cell Invasion and Salmonella Pathogenicity Island 1 Expression between Salmonella enterica Serovar Paratyphi A and Nontyphoidal S. Typhimurium. Infect. Immun. 2016, 84, 1150-1165. [CrossRef]

12. Lambert, M.A.; Smith, S.G. The PagN protein of Salmonella enterica serovar Typhimurium is an adhesin and invasin. BMC Microbiol. 2008, 8, 142. [CrossRef]

13. Cirillo, D.M.; Heffernan, E.J.; Wu, L.; Harwood, J.; Fierer, J.; Guiney, D.G. Identification of a domain in Rck, a product of the Salmonella typhimurium virulence plasmid, required for both serum resistance and cell invasion. Infect. Immun. 1996, 64, 2019-2023. [CrossRef] [PubMed]

14. Velge, P.; Wiedemann, A.; Rosselin, M.; Abed, N.; Boumart, Z.; Chausse, A.M.; Grepinet, O.; Namdari, F.; Roche, S.M.; Rossignol, A.; et al. Multiplicity of Salmonella entry mechanisms, a new paradigm for Salmonella pathogenesis. Microbiologyopen 2012, 1, 243-258. [CrossRef] [PubMed]

15. Wiedemann, A.; Mijouin, L.; Ayoub, M.A.; Barilleau, E.; Canepa, S.; Teixeira-Gomes, A.P.; Le Vern, Y.; Rosselin, M.; Reiter, E.; Velge, P. Identification of the epidermal growth factor receptor as the receptor for Salmonella Rck-dependent invasion. FASEB J. 2016, 30, 4180-4191. [CrossRef] [PubMed]

16. Heffernan, E.J.; Wu, L.; Louie, J.; Okamoto, S.; Fierer, J.; Guiney, D.G. Specificity of the complement resistance and cell association phenotypes encoded by the outer membrane protein genes rck from Salmonella typhimurium and ail from Yersinia enterocolitica. Infect. Immun. 1994, 62, 5183-5186. [CrossRef]

17. Ho, D.K.; Jarva, H.; Meri, S. Human complement factor H binds to outer membrane protein Rck of Salmonella. J. Immunol. 2010, 185, 1763-1769. [CrossRef]

18. Glaubman, J.; Hofmann, J.; Bonney, M.E.; Park, S.; Thomas, J.M.; Kokona, B.; Ramos Falcon, L.I.; Chung, Y.K.; Fairman, R.; Okeke, I.N. Self-association motifs in the enteroaggregative Escherichia coli heat-resistant agglutinin 1. Microbiology 2016, 162, 1091-1102. [CrossRef]

19. Bhargava, S.; Johnson, B.B.; Hwang, J.; Harris, T.A.; George, A.S.; Muir, A.; Dorff, J.; Okeke, I.N. Heat-resistant agglutinin 1 is an accessory enteroaggregative Escherichia coli colonization factor. J. Bacteriol. 2009, 191, 4934-4942. [CrossRef]

20. Fleckenstein, J.M.; Kopecko, D.J.; Warren, R.L.; Elsinghorst, E.A. Molecular characterization of the tia invasion locus from enterotoxigenic Escherichia coli. Infect. Immun. 1996, 64, 2256-2265. [CrossRef]

21. Fagan, R.P.; Lambert, M.A.; Smith, S.G. The hek outer membrane protein of Escherichia coli strain RS218 binds to proteoglycan and utilizes a single extracellular loop for adherence, invasion, and autoaggregation. Infect. Immun. 2008, 76, 1135-1142. [CrossRef]

22. Mancini, J.; Weckselblatt, B.; Chung, Y.K.; Durante, J.C.; Andelman, S.; Glaubman, J.; Dorff, J.D.; Bhargava, S.; Lijek, R.S.; Unger, K.P.; et al. The heat-resistant agglutinin family includes a novel adhesin from enteroaggregative Escherichia coli strain 60A. J. Bacteriol. 2011, 193, 4813-4820. [CrossRef]

23. Ghosh, S.; Chakraborty, K.; Nagaraja, T.; Basak, S.; Koley, H.; Dutta, S.; Mitra, U.; Das, S. An adhesion protein of Salmonella enterica serovar Typhi is required for pathogenesis and potential target for vaccine development. Proc. Natl. Acad. Sci. USA 2011, 108, 3348-3353. [CrossRef] [PubMed]

24. Zhang, X.L.; Tsui, I.S.; Yip, C.M.; Fung, A.W.; Wong, D.K.; Dai, X.; Yang, Y.; Hackett, J.; Morris, C. Salmonella enterica serovar typhi uses type IVB pili to enter human intestinal epithelial cells. Infect. Immun. 2000, 68, 3067-3073. [CrossRef] [PubMed]

25. Yue, M.; Han, X.; Masi, L.D.; Zhu, C.; Ma, X.; Zhang, J.; Wu, R.; Schmieder, R.; Kaushik, R.S.; Fraser, G.P.; et al. Allelic variation contributes to bacterial host specificity. Nat. Commun. 2015, 6, 8754. [CrossRef] [PubMed]

26. Guo, A.; Cao, S.; Tu, L.; Chen, P.; Zhang, C.; Jia, A.; Yang, W.; Liu, Z.; Chen, H.; Schifferli, D.M. FimH alleles direct preferential binding of Salmonella to distinct mammalian cells or to avian cells. Microbiology 2009, 155, 1623-1633. [CrossRef]

27. Hersh, D.; Monack, D.M.; Smith, M.R.; Ghori, N.; Falkow, S.; Zychlinsky, A. The Salmonella invasin SipB induces macrophage apoptosis by binding to caspase-1. Proc. Natl. Acad. Sci. USA 1999, 96, 2396-2401. [CrossRef] 
28. Hermant, D.; Menard, R.; Arricau, N.; Parsot, C.; Popoff, M.Y. Functional Conservation of the Salmonella and Shigella Effectors of Entry into Epithelial-Cells. Mol. Microbiol. 1995, 17, 781-789. [CrossRef]

29. Cunrath, O.; Meinel, D.M.; Maturana, P.; Fanous, J.; Buyck, J.M.; Saint Auguste, P.; Seth-Smith, H.M.B.; Korner, J.; Dehio, C.; Trebosc, V.; et al. Quantitative contribution of efflux to multi-drug resistance of clinical Escherichia coli and Pseudomonas aeruginosa strains. EBio Med. 2019, 41, 479-487. [CrossRef]

30. Gibson, D.G.; Young, L.; Chuang, R.Y.; Venter, J.C.; Hutchison, C.A., 3rd; Smith, H.O. Enzymatic assembly of DNA molecules up to several hundred kilobases. Nat. Methods 2009, 6, 343-345. [CrossRef]

31. Edwards, R.A.; Keller, L.H.; Schifferli, D.M. Improved allelic exchange vectors and their use to analyze 987P fimbria gene expression. Gene 1998, 207, 149-157. [CrossRef]

32. Blomfield, I.C.; McClain, M.S.; Eisenstein, B.I. Type 1 fimbriae mutants of Escherichia coli K12: Characterization of recognized afimbriate strains and construction of new fim deletion mutants. Mol. Microbiol. 1991, 5, 1439-1445. [CrossRef]

33. Harms, A.; Liesch, M.; Korner, J.; Quebatte, M.; Engel, P.; Dehio, C. A bacterial toxin-antitoxin module is the origin of inter-bacterial and inter-kingdom effectors of Bartonella. PLoS Genet. 2017, 13, e1007077. [CrossRef] [PubMed]

34. Hoiseth, S.K.; Stocker, B.D. Aromatic-dependent Salmonella typhimurium are nonvirulent and effective as live vaccines. Nature 1981, 291, 238-239. [CrossRef] [PubMed]

35. Cao, J.; Khan, A.S.; Bayer, M.E.; Schifferli, D.M. Ordered translocation of 987P fimbrial subunits through the outer membrane of Escherichia coli. J. Bacteriol. 1995, 177, 3704-3713. [CrossRef] [PubMed]

36. Nair, M.K.; De Masi, L.; Yue, M.; Galvan, E.M.; Chen, H.; Wang, F.; Schifferli, D.M. Adhesive properties of YapV and paralogous autotransporter proteins of Yersinia pestis. Infect. Immun. 2015, 83, 1809-1819. [CrossRef]

37. Schifferli, D.M.; Alrutz, M.A. Permissive linker insertion sites in the outer membrane protein of $987 \mathrm{P}$ fimbriae of Escherichia coli. J. Bacteriol. 1994, 176, 1099-1110. [CrossRef]

38. Liu, F.; Chen, H.; Galván, E.M.; Lasaro, M.A.; Schifferli, D.M. Effects of Psa and F1 on the adhesive and invasive interactions of Yersinia pestis with human respiratory tract epithelial cells. Infect. Immun. 2006, 74, 5636-5644. [CrossRef]

39. Yoshida, C.E.; Kruczkiewicz, P.; Laing, C.R.; Lingohr, E.J.; Gannon, V.P.; Nash, J.H.; Taboada, E.N. The Salmonella In Silico Typing Resource (SISTR): An Open Web-Accessible Tool for Rapidly Typing and Subtyping Draft Salmonella Genome Assemblies. PLoS ONE 2016, 11, e0147101. [CrossRef]

40. Zhang, C.; Freddolino, P.L.; Zhang, Y. COFACTOR: Improved protein function prediction by combining structure, sequence and protein-protein interaction information. Nucleic Acids Res. 2017, 45, W291-W299. [CrossRef]

41. Yang, J.; Zhang, Y. I-TASSER server: New development for protein structure and function predictions. Nucleic Acids Res. 2015, 43, W174-W181. [CrossRef]

42. Colovos, C.; Yeates, T.O. Verification of protein structures: Patterns of nonbonded atomic interactions. Protein Sci. 1993, 2, 1511-1519. [CrossRef]

43. Goswami, N.; Hussain, M.I.; Borah, P. Molecular dynamics approach to probe the antigenicity of PagN-An outer membrane protein of Salmonella Typhi. J. Biomol. Struct. Dyn. 2018, 36, 2131-2146. [CrossRef] [PubMed]

44. Yue, M.; Rankin, S.C.; Blanchet, R.T.; Nulton, J.D.; Edwards, R.A.; Schifferli, D.M. Diversification of the Salmonella fimbriae: A model of macro- and microevolution. PLoS ONE 2012, 7, e38596. [CrossRef] [PubMed]

45. Hansmeier, N.; Miskiewicz, K.; Elpers, L.; Liss, V.; Hensel, M.; Sterzenbach, T. Functional expression of the entire adhesiome of Salmonella enterica serotype Typhimurium. Sci. Rep. 2017, 7, 10326. [CrossRef] [PubMed]

46. Su, Y.C.; Halang, P.; Fleury, C.; Jalalvand, F.; Morgelin, M.; Riesbeck, K. Haemophilus Protein F Orthologs of Pathogens Infecting the Airways: Exploiting Host Laminin at Heparin-Binding Sites for Maximal Adherence to Epithelial Cells. J. Infect. Dis. 2017, 216, 1303-1307. [CrossRef]

47. Izquierdo, M.; Navarro-Garcia, F.; Nava-Acosta, R.; Nataro, J.P.; Ruiz-Perez, F.; Farfan, M.J. Identification of cell surface-exposed proteins involved in the fimbria-mediated adherence of enteroaggregative Escherichia coli to intestinal cells. Infect. Immun. 2014, 82, 1719-1724. [CrossRef] 
48. Samadder, P.; Xicohtencatl-Cortes, J.; Saldana, Z.; Jordan, D.; Tarr, P.I.; Kaper, J.B.; Giron, J.A. The Escherichia coli ycbQRST operon encodes fimbriae with laminin-binding and epithelial cell adherence properties in Shiga-toxigenic E. coli O157:H7. Environ. Microbiol. 2009, 11, 1815-1826. [CrossRef]

49. Gunn, J.S.; Belden, W.J.; Miller, S.I. Identification of PhoP-PhoQ activated genes within a duplicated region of the Salmonella typhimurium chromosome. Microb. Pathog. 1998, 25, 77-90. [CrossRef]

50. Park, S.Y.; Groisman, E.A. Signal-specific temporal response by the Salmonella PhoP/PhoQ regulatory system. Mol. Microbiol. 2014, 91, 135-144. [CrossRef]

51. Golubeva, Y.A.; Ellermeier, J.R.; Cott Chubiz, J.E.; Slauch, J.M. Intestinal Long-Chain Fatty Acids Act as a Direct Signal To Modulate Expression of the Salmonella Pathogenicity Island 1 Type III Secretion System. mBio 2016, 7, e02170-15. [CrossRef]

52. Kouzi-Koliakos, K.; Koliakos, G.G.; Tsilibary, E.C.; Furcht, L.T.; Charonis, A.S. Mapping of three major heparin-binding sites on laminin and identification of a novel heparin-binding site on the B1 chain. J. Biol. Chem. 1989, 264, 17971-17978.

53. Lambert, M.A.; Smith, S.G. The PagN protein mediates invasion via interaction with proteoglycan. FEMS Microbiol. Lett. 2009, 297, 209-216. [CrossRef] [PubMed]

54. De Masi, L.; Yue, M.; Hu, C.; Rakov, A.V.; Rankin, S.C.; Schifferli, D.M. Cooperation of Adhesin Alleles in Salmonella-Host Tropism. mSphere 2017, 2. [CrossRef] [PubMed]

55. Hayden, H.S.; Matamouros, S.; Hager, K.R.; Brittnacher, M.J.; Rohmer, L.; Radey, M.C.; Weiss, E.J.; Kim, K.B.; Jacobs, M.A.; Sims-Day, E.H.; et al. Genomic Analysis of Salmonella enterica Serovar Typhimurium Characterizes Strain Diversity for Recent U.S. Salmonellosis Cases and Identifies Mutations Linked to Loss of Fitness under Nitrosative and Oxidative Stress. MBio 2016, 7, e00154. [CrossRef] [PubMed]

(C) 2020 by the authors. Licensee MDPI, Basel, Switzerland. This article is an open access article distributed under the terms and conditions of the Creative Commons Attribution (CC BY) license (http://creativecommons.org/licenses/by/4.0/). 